\title{
Diabetes mellitus, síndrome metabólica e risco de queda: um estudo seccional com
}

\section{idosos da comunidade}

\author{
Diabetes Mellitus, metabolic syndrome and fall risk: a cross-sectional study with community- \\ dwelling elderly \\ Diabetes Mellitus, síndrome metabólico y riesgo de caídas: un estudio transversal con ancianos que \\ viven en la comunidad
}

Greiciane da Silva Rocha

ORCID: https://orcid.org/0000-0002-1636-7179

Universidade Federal do Acre, Brasil

E-mail: greiciane.rocha@ufac.br

Catherine de Farias Sussuarana

ORCID: https://orcid.org/0000-0003-1994-8961

Universidade Federal do Acre, Brasil

E-mail: catherinesussuarana@gmail.com

Tábatta Renata Pereira de Brito

ORCID: https://orcid.org/0000-0001-9466-2993

Universidade Federal de Alfenas, Brasil

E-mail: tabatta_renata@hotmail.com

Francimar Leão Jucá

ORCID: https://orcid.org/0000-0002-3612-2355

Ozônio Vida Clínica, Brasil

E-mail: francilj1@gmail.com

Rozilaine Redi Lago

ORCID: https://orcid.org/0000-0002-1383-0463 Universidade Federal do Acre, Brasil E-mail: rozilaine.lago@ufac.br

Alice Helena de Souza Paulino

ORCID: https://orcid.org/0000-0003-3906-8259

Universidade Federal de Alfenas, Brasil

E-mail: alicehpaulino@gmail.com

Valgerlângela Maria Sousa da Silva

ORCID: https://orcid.org/0000-0001-6948-0530

Secretaria Municipal de Saúde de Rio Branco, Brasil E-mail: valger.angela@gmail.com

\begin{abstract}
Resumo
O artigo tem o objetivo de comparar a associação entre síndrome metabólica e diabetes com o risco de quedas em idosos da comunidade. Para alcançar os objetivos optou-se por um estudo quantitativo com delineamento transversal do tipo analítico com indivíduos de 60 anos ou mais, não institucionalizados, residentes em área urbana do município de Rio Branco/Acre. O risco elevado de quedas correspondeu a 77 idosos do sexo masculino (43,4\%), idosos independente do sexo com 80 anos ou mais de idade $(70,7 \%)$, apresentando sintomas depressivos $(53,6 \%)$ e dependentes de auxílio para a realização de atividades básicas de vida diária $(51,7 \%)$. No modelo para síndrome metabólica houve associação com sintomas depressivos, atividades instrumentais de vida diária e faixa etária, sendo que os idosos com sintomas depressivos, dependência para as atividades instrumentais de vida diária e faixa etária de 70 a 79 anos e 80 anos e mais obtiveram risco elevado de quedas. No modelo com diabetes, há um aumento na chance no risco de quedas, juntamente com sintomas depressivos, atividades instrumentais de vida diária e faixa etária independente das atividades básicas de vida diária, escolaridade e do declínio cognitivo. Devido ao estado de saúde comprometido pelo descontrole do diabetes, ganho e perda de peso excessivos, ingestão alimentar insuficiente, o idoso se torna suscetível à variação dos níveis séricos de tolerância à glicose e aquisição de sarcopenia contribuindo para futuros problemas como osteoporose, quedas, fraturas e perda na independência ou mobilidade.
\end{abstract}

Palavras-chave: Síndrome metabólica; Diabetes Mellitus; Acidentes por quedas; Idoso.

\section{Abstract}

This is the objective of comparing the association between metabolic syndrome and diabetes as a risk of remaining em elderlys in the community. To achieve the objectives, a quantitative study was chosen with a cross-sectional design of 
an analytical type with individuals aged 60 years or older, not institutionalized, residents in the urban area of the municipality of Rio Branco / Acre. The high risk of falls corresponded to 77 male persons (43.4\%), persons independent of the sex aged 80 years or older $(70.7 \%)$, showing depressive symptoms $(53.6 \%)$ and dependent on assistance for the performance of basic daily life activities $(51.7 \%)$. Non-model for metabolic syndrome there was association with depressive symptoms, instrumental activities of daily life and age group, being that those with depressive symptoms, dependence for instrumental activities of daily life and age group from 70 to 79 years and 80 years and most of had high risk of falling. In the diabetes model, there is an increased chance of risk of falls, along with depressive symptoms, instrumental activities of daily living and age group independent of basic activities of daily living, education and cognitive decline. Due to the health status compromised by the lack of diabetes control, excessive weight gain and loss, insufficient food intake, the elderly becomes susceptible to variation in serum glucose tolerance levels and sarcopenia acquisition, contributing to future problems such as osteoporosis, falls, fractures and loss of independence or mobility.

Keywords: Metabolic syndrome; Diabetes Mellitus; Accidental falls; Aged.

\section{Resumem:}

El artículo tiene como objetivo comparar la asociación entre síndrome metabólico y diabetes con el riesgo de caídas en personas mayores de la comunidad. Para lograr los objetivos se eligió un estudio cuantitativo con un diseño analítico transversal, con personas no institucionalizadas de 60 años o más, residentes en un area urbana de la ciudad de Rio Branco/Acre. El alto riesgo de caídas correspondió a 77 ancianos varones (43,4\%), ancianos independientemente del sexo de 80 años o más (70,7\%), con síntomas depresivos $(53,6 \%)$ y dependientes de asistencia para realizar actividades básicas de la vida diaria (51,7\%). En el modelo de síndrome metabólico, hubo asociación con síntomas depresivos, actividades instrumentales de la vida diaria y grupo de edad, y ancianos con síntomas depresivos, dependencia para las actividades instrumentales de la vida diaria y grupo de edad de 70 a 79 años y 80 años y sobre obtenido alto riesgo de caídas. En el modelo de diabetes, existe una mayor probabilidad de riesgo de caídas, junto con síntomas depresivos, actividades instrumentales de la vida diaria y grupo de edad independiente de las actividades básicas de la vida diaria, educación y declinación cognitivo. Debido al estado de salud comprometido por la diabetes no controlada, el aumento y la pérdida excesiva de peso, la ingesta insuficiente de alimentos, los ancianos se vuelven susceptibles a variaciones en los niveles séricos de tolerancia a la glucosa y adquisición de sarcopenia, contribuyendo a problemas futuros como osteoporosis, caídas, fracturas y pérdida de independencia o movilidad.

Palabras clave: Síndrome metabólico; Diabetes Mellitus; Accidentes por caídas; Anciano.

\section{Introdução}

A queda da fertilidade e o crescente aumento na expectativa de vida da população impactaram diretamente na dinâmica da transição demográfica ao redor do mundo. O expressivo envelhecimento populacional implica em preocupações e grandes desafios para o século XXI, devido às características fisiológicas, psicológicas e sociais que esse processo envolve (Who, 2017; Kehoe, Walton \& Flynn, 2019).

Estima-se que entre 2050 e 2070, com a redução gradativa da mortalidade, a expectativa de vida de países desenvolvidos, como França e Japão, possa chegar aos 100 anos (Vaupel et al., 2021). No Brasil, segundo o Instituto Brasileiro de Geografia e Estatística (IBGE, 2021), uma contribuição significativa para o processo de envelhecimento populacional deve ocorrer a partir de 2047, uma vez que a projeção é de um aumento de 43,2\%, em 2018, para 173,5\% em 2060.

A intensificação do declínio funcional, que ocorre por volta dos 60 anos, conduz ao desenvolvimento de condições características dessa faixa etária (Shlisky et al., 2017). Dentre as principais, destaca-se a ocorrência de quedas, que são eventos frequentes em idosos e implicam em perda da autonomia por deficiências e lesões, sendo umas das principais causas de mortalidade entre esses indivíduos (Lee et al., 2020). Intimamente relacionadas com a postura e a marcha, as quedas costumam ser consequências da aquisição de morbidades a citar entre essas a diabetes e síndrome metabólicas que envolvidas com o desequilíbrio em múltiplos sistemas, são capazes de comprometer a habilidade de compensação do indivíduo (Canuto et al, 2020).

Quanto as morbidades associadas e precursoras da queda em idosos, a síndrome metabólica e a diabetes são prevalentes entre os idosos e reponsáveis pelo desíquilibrio sistêmico levando o compromentimento da estabilidade postural. Contudo, vale destacar que pacientes com diagnóstico de diabetes melittus tipo 2 podem incrementar o quadro de síndrome 
metabólica (Gurka et al., 2018), e essa forte relação pode se intensificar quanto mais numeroso forem os componentes da síndrome (Simão et al., 2013).

Assim, a síndrome metabólica, caracterizada como um conjunto de desordens metabólicas, envolve comorbidades como dislipidemia, obesidade abdominal e hipertensão arterial, que culminam em maior risco para o desenvolvimento de doenças cardiovasculares e diabetes. Com grande prevalência em idosos, a síndrome metabólica desencadeia uma piora cognitiva-funcional e dificuldade de mobilidade, representando uma vulnerabilidade aumentada para o risco de quedas (Liaw et al., 2016; Tamura et al., 2020).

Associado ao próprio processo de envelhecimento, lesões e à polifarmácia, o diabetes mellitus, de forma isolada, também é descrito como um importante fator no risco elevado do comprometimento do equilíbrio e distúrbios da marcha. Um dos motivos é o acometimento pela neuropatia diabética, que leva a um déficit sensório-motor, aumentando o risco de quedas, fraturas e morte (Gu \& Dennis, 2017; Rinkel et al., 2019). Ainda, os distúrbios metabólicos induzidos pelo diabetes, podem provocar alterações na microarquitetura óssea, remodelação óssea e células osteogênicas (Wallander et al., 2017).

Nesse sentido, por se ter poucos estudos com um abordagem de destaque da diabetes e síndrome metabólica como efeito precurssor nas quedas em idosos é que se propôs o estudo em tela para analisar a associação de síndrome metabólica e diabetes com o risco de queda em idosos da comunidade, a fim de direcionar medidas preventivas, com um rastreio diagnóstico preciso e precoce para uma intervenção terapeutica efetiva e de melhoria na qualidade de vida dos idosos.

\section{Metodologia}

Esse projeto é um recorte do estudo intitulado "Estudo multicêntrico sobre a associação entre fatores de risco cardiometábolicos e de quedas, apoio social e multimorbidade em idosos", financiado pela FAPAC (Edital 007/20217) do programa de pesquisa para o SUS: Gestão compartilhada em saúde - PPSUS/AC, aprovado pelo Comitê de Ética em Pesquisa do Hospital das Clínicas do Acre - HCA/FUNDHACRE em 06 de outubro de 2017, sob o parecer 2.319.053 e CAAE: 76889417.3.1001.5009. O projeto seguiu os preceitos éticos para investigação com envolvimento de seres humanos, especialmente os que estão descritos na resolução n.466/2012 (Brasil, 2012).

Trata-se de um estudo quantitativo com delineamento transversal (Polit \& Beck, 2019) com indivíduos de 60 anos ou mais, não institucionalizados, residentes em área urbana do município de Rio Branco. Foi planejada amostra probabilística de 441 idosos em Rio Branco. Esse tamanho de amostra foi obtido considerando-se a estimação de proporções da ordem de 0,50 com erro de amostragem de 10\%, nível de 95\% de confiança e efeito de delineamento de 1,17.

A população com 60 anos ou mais de Rio Branco em 2015 (último ano disponível com estimativa populacional por município e idade no DATASUS) era de 24.043 indivíduos (MS, 2021). A seleção aleatória da amostra foi realizada a partir dos cadastros de idosos no Sistema G-MUS - Gestão Municipal de Saúde, por meio do Microsoft Office Excel. Para realização do sorteio utilizou-se a lista de idosos cadastrados em novembro de 2018, que totalizava 22.370 idosos, ou seja, $89,3 \%$ da população com 60 anos ou mais utilizada para o cálculo do tamanho da amostra.

Os critérios de inclusão foram: idade de 60 anos ou superior que concordassem com a participação mediante assinatura do TCLE. O critério de exclusão foi apresentar condições neurológicas e cognitivas que impossibilitem o idoso responder aos questionários. A coleta de dados foi realizada em duas etapas, durante a primeira procedeu-se a entrevista pessoal e avaliação da composição corporal (antropometria), segunda etapa, a partir de agendamento prévio com idoso e familiares e/ou cuidadores obteve-se a coleta de sangue.

Objetivando a realização da entrevista, foi agendado um horário para a aplicação do questionário com cada idoso participante, contendo questões referentes aos seguintes blocos: Bloco A, constando a identificação do idoso e características 
sociodemográficas. Bloco B, avaliação da cognição por meio do Mini Exame do Estado mental e Escala de Depressão Geriátrica. E Bloco C, contendo informações sobre Estado Geral de Saúde.

Por conseguinte, o Bloco D, estipulando sobre a capacidade funcional para atividades básicas diárias. Bloco E, informações sobre o apoio social recebido. Bloco F, acerca dos tipos e práticas de atividades físicas. Bloco G, aspectos nutricionais envolvendo medidas antropométricas: tipo, qualidade e quantidade de consumo alimentar (recordatório de 24 horas). Bloco H, ocorrências e tipos de quedas. E Bloco I, resultando a análise bioquímica.

Para esse recorte do estudo foi utilizado como variável dependente o risco de queda pelo teste de Timed Up and Go (TUG). Cada participante realizava o trajeto (3 metros em linha reta) com seu próprio calçado e quaisquer auxílios da macha que já fizesse uso. O tempo para realizar todo o trajeto foi registrado em segundos. O ponto de corte seguido foi do estudo de Alexandre et al. (2012) de $\geq 12,47$ seg para alto risco de queda e $<12,47$ para baixo risco.

As variáveis independentes foram sexo, faixa etária, anos de estudo, situação conjugal, renda familiar, arranjo domiciliar, declínio cognitivo, sintomas depressivos, multimorbidade, polifarmácia, ABVD, AIVD, síndrome metabólica, diabetes autorreferida. Para o diagnóstico da síndrome metabólica, utilizou-se os critérios pré-estabelecidos para população adulta da Organização mundial de saúde (OMS), do National Cholesterol Education Program's Adult Treatment Panel III (NCEP-ATP III) e da International Diabetes Federation (IDF) (Alberti et al., 2009).

Os dados foram digitados no programa Excel e analisados no Software Stata, versão 15.0. Foram estimadas as proporções na análise descritiva dos dados. Para as análises de associação, utilizou-se regressão logística múltipla. A magnitude da associação foi estimada pela razão de chances (OR) bruta e ajustada. As variáveis que apresentaram valor de $\mathrm{p}$ menor que 0,20 na análise univariada foram incluídas nos modelos múltiplos, sendo construídos dois modelos: modelo A para síndrome metabólica e modelo B para diabetes. Adotou-se o nível de significância de 5\%.

\section{Resultados}

Foram acompanhados 441 idosos com faixa etária de 60 anos, desses idosos maior parte se concentrou no público feminino e na faixa etária de 60 e mais. Na análise univariada um percentual em cada categoria de variável se apresentou vulnerável ao evento da queda. Entre os idosos do sexo masculino $59(43,4 \%)$ e o feminino $114(37,4)$ apresentaram o risco de cair. Quanto as demais variáveis, no público de 80 e mais 41(70,7\%), idosos com declínio cognitivo 57 (55,9\%), sintomas depressivos $82(53,6 \%)$, dependente para as atividades básica de vida diária 31 (51,7\%) e diabetes com 53(53,0\%) contribuíram para a predisposição dos idosos a queda (Tabela 1). 
Tabela 1 - Univariada da associação entre risco de quedas e variáveis socioeconômicas e de saúde. Rio Branco/AC, 2019 (n= 441).

\begin{tabular}{|c|c|c|c|c|c|c|}
\hline \multirow{3}{*}{ Variáveis } & \multicolumn{4}{|c|}{ Risco de Queda } & \multirow{3}{*}{$\mathbf{O R}^{\mathrm{a}}$} & \multirow{3}{*}{$\mathbf{p}$} \\
\hline & \multicolumn{2}{|c|}{ Não } & \multicolumn{2}{|c|}{ Sim } & & \\
\hline & $\mathbf{n}$ & $\%$ & $\mathbf{n}$ & $\%$ & & \\
\hline \multicolumn{7}{|l|}{ Sexo } \\
\hline Masculino & 77 & 56.6 & 59 & 43.4 & 1.00 & \\
\hline Feminino & 191 & 62.6 & 114 & 37.4 & 1.28 & 0.233 \\
\hline \multicolumn{7}{|l|}{ Faixa Etária } \\
\hline 60 a 69 anos & 152 & 72.7 & 57 & 27.3 & 1.00 & \\
\hline 70 a 79 anos & 99 & 56.9 & 75 & 43.1 & 2.02 & 0.001 \\
\hline 80 anos ou mais & 17 & 29.3 & 41 & 70.7 & 6.43 & 0.000 \\
\hline \multicolumn{7}{|l|}{ Anos de Estudo } \\
\hline$\leq 4$ anos & 241 & 59,8 & 162 & 40,2 & 1.00 & \\
\hline$>4$ anos & 26 & 72,2 & 10 & 27,8 & 0.57 & 0.148 \\
\hline \multicolumn{7}{|l|}{ Situação Conjugal } \\
\hline Sem companheiro & 155 & 61.8 & 96 & 38.3 & 1.00 & \\
\hline Com companheiro & 113 & 59.8 & 76 & 40.2 & 1.08 & 0.676 \\
\hline \multicolumn{7}{|l|}{ Renda Familiar } \\
\hline$\leq 1$ salário-mínimo & 70 & 60.9 & 45 & 39.1 & 1.00 & \\
\hline$>1$ salário mínimo & 183 & 62.0 & 112 & 38.0 & 0.95 & 0.828 \\
\hline \multicolumn{7}{|l|}{ Arranjo Domiciliar } \\
\hline Não mora sozinho & 229 & 61.9 & 141 & 38.1 & 1.00 & \\
\hline Mora sozinho & 39 & 54.9 & 32 & 45.1 & 1.33 & 0.272 \\
\hline \multicolumn{7}{|l|}{ Declínio Cognitivo } \\
\hline Não & 212 & 66.3 & 108 & 33.8 & 1.00 & \\
\hline Sim & 45 & 44.1 & 57 & 55.9 & 2.48 & 0.000 \\
\hline \multicolumn{7}{|l|}{ Sintomas Depressivos } \\
\hline Não & 180 & 68.7 & 82 & 31.3 & 1.00 & \\
\hline Sim & 71 & 46.4 & 82 & 53.6 & 2.53 & 0.000 \\
\hline \multicolumn{7}{|l|}{ Multimorbidade } \\
\hline Não & 36 & 66.7 & 18 & 33.3 & 1.00 & \\
\hline Sim & 222 & 59.4 & 152 & 40.7 & 1.17 & 0.306 \\
\hline \multicolumn{7}{|l|}{ Polifarmácia } \\
\hline Não & 181 & 62.2 & 110 & 37.8 & 1.00 & \\
\hline Sim & 64 & 55.7 & 51 & 44.4 & 1.31 & 0.225 \\
\hline \multicolumn{7}{|l|}{$\mathrm{ABVD}^{\mathrm{c}}$} \\
\hline Independente & 239 & 62.7 & 142 & 37.3 & 1.00 & \\
\hline Dependente & 29 & 48.3 & 31 & 51.7 & 1.79 & 0.035 \\
\hline \multicolumn{7}{|l|}{ AIVD $^{d}$} \\
\hline Independente & 138 & 74.2 & 48 & 25.8 & 1.00 & \\
\hline Dependente & 130 & 51.2 & 124 & 48.8 & 2.74 & 0.000 \\
\hline \multicolumn{7}{|l|}{ Síndrome Metabólica } \\
\hline Não & 135 & 63.4 & 78 & 36.6 & 1.00 & \\
\hline Sim & 133 & 58.3 & 95 & 41.7 & 1.24 & 0.278 \\
\hline \multicolumn{7}{|l|}{ Diabetes } \\
\hline Não & 221 & 64.8 & 120 & 35.2 & & \\
\hline Sim & 47 & 47.0 & 53 & 53.0 & 2.08 & 0.002 \\
\hline
\end{tabular}

${ }^{\mathrm{a}} \mathrm{OR}=$ Odds ratio; ${ }^{\mathrm{b}}$ Salário-mínimo vigente $=\mathrm{R} \$ 998,00 ;{ }^{\mathrm{c}} \mathrm{ABVD}=$ Atividades Básicas de Vida Diária; ${ }^{\mathrm{d}}$ AIVD $=$ Atividades Instrumentais de Vida Diária. Fonte: Pesquisa de campo.

Nos modelos de regressão logística múltipla para o risco de quedas foram relacionados dados quanto à presença de síndrome metabólica (modelo A) e diabetes mellitus (modelo B). No modelo para síndrome metabólica houve associação com sintomas depressivos, atividades instrumentais de vida diária e faixa etária, sendo que quem tem sintomas depressivos, dependência para as atividades instrumentais de vida diária e faixa etária de 70 anos ou mais apresenta maior chance de risco elevado de queda. No modelo com diabetes mellitus, essa comorbidade se apresentou como um fator associado ao risco de quedas. Há um aumento na chance no risco de quedas, juntamente com sintomas depressivos, atividades instrumentais de vida diária e faixa etária independente das atividades básicas de vida diária, escolaridade e do declínio cognitivo (Tabela 2). 
Tabela 2 - Modelos de regressão logística múltipla para o risco de quedas. Rio Branco/AC, 2019 (n= 441).

\begin{tabular}{|c|c|c|c|c|}
\hline & \multicolumn{2}{|c|}{ Modelo A } & \multicolumn{2}{|c|}{ Modelo B } \\
\hline & OR $^{\mathbf{a}}{ }_{\text {Ajustada }}$ & IC95\% ${ }^{b}$ & OR $_{\text {Ajustada }}$ & IC95\% \\
\hline Síndrome Metabólica & 1.15 & $0.73 ; 1.81$ & & \\
\hline Não & & & - & - \\
\hline Sim & & & - & - \\
\hline Diabetes & & & 2.11 & $1.23 ; 3.61$ \\
\hline Não & - & - & & \\
\hline Sim & - & - & & \\
\hline Sintomas Depressivos & 2.64 & $1.66 ; 4.20$ & 2.59 & $1.61 ; 4.13$ \\
\hline \multicolumn{5}{|l|}{ Não } \\
\hline \multicolumn{5}{|l|}{ Sim } \\
\hline Declínio Cognitivo & 1.46 & 0,$85 ; 2.50$ & 1.44 & 0,$84 ; 2.48$ \\
\hline \multicolumn{5}{|l|}{ Não } \\
\hline \multicolumn{5}{|l|}{ Sim } \\
\hline AIVD $^{c}$ & 2.20 & $1.35 ; 3.58$ & 2.20 & $1.35 ; 3.59$ \\
\hline \multicolumn{5}{|l|}{ Independente } \\
\hline \multicolumn{5}{|l|}{ Dependente } \\
\hline \multicolumn{5}{|l|}{ Faixa Etária } \\
\hline \multicolumn{5}{|l|}{60 a 69 anos } \\
\hline 70 a 79 anos & 2.05 & $1.27 ; 3.32$ & 2.07 & $1.28 ; 3.35$ \\
\hline 80 anos ou mais & 5.63 & $2.61 ; 12.2$ & 6.10 & $2.79 ; 13.3$ \\
\hline $\mathbf{A B V D}^{\mathrm{d}}$ & 1.25 & $0.64 ; 2.43$ & 1.16 & $0.59 ; 2.28$ \\
\hline \multicolumn{5}{|l|}{ Independente } \\
\hline \multicolumn{5}{|l|}{ Dependente } \\
\hline Anos de Estudo & 0.86 & $0.34 ; 2.22$ & 0.81 & $0.32 ; 2.12$ \\
\hline$>4$ anos & & & & \\
\hline$\leq 4$ anos & & & & \\
\hline
\end{tabular}

${ }^{\mathrm{a}} \mathrm{OR}=$ Odds ratio; ${ }^{\mathrm{b}} \mathrm{IC} 95 \%$ = Intervalo de Confiança de 95\%; ${ }^{\mathrm{c}} \mathrm{ABVD}=$ Atividades Básicas de Vida Diária; ${ }^{\mathrm{A}} \mathrm{IVD}=$ Atividades Instrumentais de Vida Diária. Fonte: Pesquisa de campo.

\section{Discussão}

O estudo em tela, mostrou uma maior participação do público feminino na amostra, o que está atrelado ao processo de envelhecimento com uma maior concentração do público feminino (Souza et al.,2021), além do fato que as mulheres buscam o serviço saúde com mais frequência em relação aos homens, e o uso e acesso ao serviço são fatores essenciais para uma melhor qualidade de vida dos idosos (Louvison et al.,2008).

Além de compor o sexo feminino há uma maior predisposição para quedas entre o público de 80 e mais, estudos apontam que o processo de envelhecimento com acréscimo de idade bem explica as ocorrências de quedas (Siqueira et al.,2011; Prato et al., 2017), com a presença de fraqueza muscular, alterações posturais, articulares, comprometimento das habilidades motoras, alterações de marcha, estabilidade postural, déficit de equilíbrio, osteoporose e perda da massa magra (Gasparotto, Falsarella \& Coimbra, 2014; Gotzmeister, Zecevic \& Salmoni, 2015).

As mulheres são bem mais predispostas ao agravo de queda e tal condição está relacionado a exposição as frequentes atividades domésticas, alterações clínicas, hormonais, menor força muscular e algum adoecimento crônico, além de vivência sem companheiro, baixa escolaridade, uso de medicamentos e idade avançada (Bchele et al.,2014; Nascimento, 2017; Xavier \& Trindade, 2018). Além disso, essas têm uma maior sobrevida frente ao sexo masculino e maior prevalência de doenças crônicas (Prato et al., 2017).

O processo de envelhecimento colabora para o aumento do declínio funcional do organismo de indivíduos idosos, ocasionando resultados clínicos e sociodemográficos desfavoráveis. Quando os fatores contribuintes para a restrição do desempenho funcional são associados à presença de síndrome metabólica e diabetes mellitus, estes, causam impactos universais negativos que necessitam da implementação de mudanças comportamentais (Dias et al., 2020) 
Considerando os resultados desse estudo, evidencia-se que um idoso com 70 anos ou mais que apresenta, em conjuntura, a síndrome metabólica (SM), presença de sintomas depressivos e que tenha limitações em atividades instrumentais de vida diária, se enquadra no grupo de idosos mais suscetíveis ao risco de quedas em decorrência destas limitações. A presença de síndrome metabólica é representada por um grupo de fatores de risco cardiometabólicos que incluem obesidade abdominal combinada com elevação de pressão arterial, glicemia de jejum e triglicerídeos, além de redução do nível de lipoproteína de alta densidade (HDL). Com isso, a SM expressa um conjunto de disfunções fisiopatológicas que são contribuintes para o desenvolvimento de aterosclerose e diabetes mellitus tipo 2 (Costa et al., 2021).

Com isso, a SM é considerada um importante problema de saúde pública devido a coexistência de três ou mais fatores de risco cardiometabólicos, como a resistência à insulina ou hiperglicemia, obesidade central, hipertensão arterial sistêmica, hipertrigliceridemia, doenças renais e osteoarticulares. O que evidencia a importância do conhecimento dos fatores agravantes e contribuintes para as doenças crônicas (Silva et al., 2019; Cavalcante, 2021).

$\mathrm{O}$ diabetes mellitus está fortemente associado com o excesso de peso e sedentarismo, tornando necessário para a manutenção da saúde a prática regular de exercícios físicos e uma dieta nutricional que vise a diminuição ou manutenção adequada do peso corporal de forma a contribuir ao controle glicêmico e reduzir as complicações associadas com a patologia, como hipertensão arterial sistêmica e dislipidemias (Flor et al., 2017).

O Brasil foi considerado, no ano de 2015, o quarto país do mundo com mais adultos com diabetes mellitus, apresentando uma população de 14,3 milhões de diabéticos. De acordo com a International Diabetes Federation (IDF) (Alberti, et al, 2009), estima-se que a população mundial de diabéticos atinja em 2040 um total de 642 milhões de pessoas, considerando que 75\% destas sejam residentes de países de baixa e média renda (Paiva et al., 2019).

Diversos fatores são responsáveis pela diminuição da qualidade de vida do idoso diagnosticado com diabetes mellitus, dentre os quais recebem destaque nos resultados deste estudo a faixa etária elevada, presença de sintomas depressivos, maus hábitos e dependência de um auxiliador para realizar suas atividades instrumentais de vida diária, pois afetam o estado físico, psíquico, funcional e o bem-estar geral do indivíduo (Paiva et al., 2019).

Além disso, o idoso com hábitos desfavoráveis à saúde contribui para o ganho de peso corporal e se torna suscetível à obesidade que é um critério maior para o diabetes. Com o processo de envelhecimento decorrente de menor tolerância à glicose, o idoso se torna propício ao risco de complicações, demanda para procedimentos complexos, aumento da taxa de permanência e mortalidade hospitalar e elevado risco de quedas (Oliveira et al., 2020).

A manutenção da capacidade funcional é um fator primordial para a independência e autonomia do idoso. Postulando a necessidade do tratamento de multimorbidades e controle dos fatores de riscos que aumentam a prevalência de quedas por idosos acometidos com síndrome metabólica e diabetes mellitus, de modo a contribuir positivamente à evolução do quadro de saúde da pessoa mais velha e aumentar sua expectativa de vida (Grosser et al., 2020).

Com isso, o profissional de saúde deve considerar a tomada de decisões oportunas para a melhora da qualidade de vida do idoso, tendo em vista suas preferências, prognósticos e comorbidades. De modo que o paciente receba uma assistência qualificada, com comunicação colaborativa e contínua, definição de metas entre os membros da equipe, com abordagens integradas e direcionadas para o cuidado centrado na pessoa (ADA, 2020).

\section{Conclusão}

Em virtude do comprometimento da saúde decorrente da presença de síndrome metabólica e descontrole do Diabetes Mellitus, o idoso se torna suscetível à variação dos níveis séricos de tolerância à glicose e aquisição de sarcopenia. Contribuindo, assim, para futuros problemas como osteoporose, quedas, fraturas, perda da independência ou mobilidade, disfunções cognitivas e risco elevado de morbimortalidade. 
Diante destas condições, é fundamental o controle dos fatores de risco associados com a síndrome metabólica e desenvolvimento do diabetes mellitus, capazes de elevar a prevalência de quedas por idosos. Tais como a introdução de hábitos de vida saudáveis como dieta nutritiva balanceada e atividades físicas regulares, a fim de diminuir o risco de resistência à insulina e agravos predeterminantes das patologias abordadas.

Conclui-se que o acúmulo de sinais e sintomas, associados à síndrome metabólica e diabetes mellitus, implica riscos importantes à qualidade de vida do idoso. Sendo estes decorrentes do desequilíbrio de habilidades metabólicas compensatórias que favorecem o risco de quedas por crédito do envelhecimento normal e patológico, o que torna imprescindível o estabelecimento da assistência qualificada durante o cuidado geriátrico.

Quanto as limitações o estudo em tela apresentou algumas condições, as quais podemos citar o uso da variável diabetes como uma condição autorreferida e não pelo diagnóstico médico comprovado, além de não poder comprovar causalidade em virtude de o desenho do estudo ter um desenho transversal. O estudo só foi possível ser feito com idosos cadastrados no sistema G-MUS da secretaria municipal de saúde, em decorrências das análises laboratoriais terem sido realizadas no laboratório do município e necessitar do cadastro do idoso no sistema de saúde municipal.

Recomenda-se que novos estudos sejam feitos partindo do diagnóstico médico de diabetes mellitus, de acompanhamento do idoso, na mensuração dos eventos da queda e de seus fatores de risco pelo Short Physycal Perfomance Battery (SPPB) e Quick Screen Clinical Falls Risk Assessment (QuickScreen), com um público de idosos maior e não só dos adscritos no sistema da secretaria de saúde do município.

\section{Agradecimentos}

Agradecemos o financiamento da pesquisa pelo programa pesquisa para o SUS: Gestão compartilhada em saúde PPSUS/AC. Chamada FAPAC-SESACRE-Decit/SCTIE/MS-CNPq sob processo nº 33376.512.21332.21092017.

\section{Referências}

ADA. American Diabetes Association. (2020). Standards of Medical Care in Diabetes: Abridged for Primary Care Providers. Clinical Diabetes. 38 (1) 10-38. $10.2337 / \mathrm{cd} 20$-as0

Albert, K., Eckel, R., Grundy, S., Zimmet, P., Cleeman, J., \& Donato, K. (2009). Harmonizing the metabolic syndrome: a joint interim statement of the International Diabetes Federation Task Force on Epidemiology and Prevention. International Diabetes Federation Task Force on Epidemiology and Prevention; Hational Heart, Lung, and Blood Institute; American Heart Association; World Heart Federation; International Atherosclerosis Society; International Association for the Study of Obesity; National Heart, Lung, and Blood Institute; American Heart Association; World Heart Federation; International Atherosclerosis Society; and International Association for the Study of Obesity. Circulation. 20;120(16):1640-5. 10.1161/CIRCULATIONAHA.109.192644

Alexandre, T.S., Meira, D.M., Rico, N. C., \& Mizuta, S. K. (2012). Accuracy of timed up and go test for screening risk of falls among Community-dwelling elderly. Rev. Brasileira de Fisioterapia. 16(5). 381-8. 10.1590/s1413-35552012005000041

Brasil. (2012). Resolução no 466, de 12 de dezembro de 2012. Dispõe sobre diretrizes e normas regulamentadoras de pesquisas envolvendo seres humanos., Diário Oficial da União.

Büchele, G., Becker, C., Cameron, I., Konig, H., Robinovitch, S., \& Rapp, K. (2014). Preditors of serious consequences of falls in residential aged care: analysis of more than 70,000 falls from residents of Bavarian nursing homes. Journal of the American Medical Directors Association. 15(8)559-563. 10.1016/j.jamda.2014.03.015

Canuto, C. P. A. S., Oliveira, L. P. B. A., Medeiros, M. R. S., \& Barros, W. C. T. S. (2020). Segurança do paciente idoso hospitalizado: Uma análise do risco de quedas. Rev. da Escola De Enfermagem da USP. 10.1590/S1980-220X2018054003613

Cavalcante, G. (2021). Principais fatores associados à Síndrome Metabólica em Idosos. Revista Multidisciplinar em Saúde. 2(1), 6. 10.51161/rems/653

Costa, M., Lima, L., Silva, I., Rehem, T., Funghetto, S., \& Stival, M. (2021). Risco cardiovascular aumentado e o papel da síndrome metabólica em idosos hipertensos. Esc. Anna Nery. 10.1590/2177-9465-EAN-2020-0055

Dias, V., Carlos, A., Lemos, A., Filho, B., Perracini, M., \& Souza, A. (2020). Fatores associados ao desempenho funcional de idosos com diabetes mellitus tipo 2. Rev. Pesquisa, Sociedade e Desenvolvimento. 9(11), 5. 10.33448/rsd-v9i11.10185 
Flor, L., \& Campos, M. (2017). Prevalência de diabetes mellitus e fatores associados na população adulta brasileira: evidências de um inquérito de base populacional. Rev. Brasileira de Epidemiologia. 20(1), 16. 10.1590/1980-5497201700010002

Gasparotto, L., Falsarella, G., \& Coimbra, A. (2014). Falls in elderly: basics concepts and updates of research in health. Rev. Brasileira de Geriatria $e$ Gerontologia.17(1): 201-9. 10.1590/S1809-98232014000100019

Gotzmeister, D., Zecevic, A., Klinger, L., \& Salmoni, A. (2015). People are getting lost a little bit: systemic factors that contribute to falls in Communitydwelling octogenarians. Canadian Journal on Aging. 34(3):397-410. 10.1017/S071498081500015X

Grosser, R., Silva, C., Kleber, P., Paula, G., Restelatto, M; \& Cetolin, S. et al. (2020). Síndrome metabólica em idosos: relação com multimorbidade e capacidade funcional/ Metabolic syndrome in the elderly: relationship with multimorbity and functional capacity. Brasilian Journal of Health Review. 10.34119/bjhrv3n4-254

Gurka, M.J., Filipp, S.L., Pearson, T.A., \& DeBoer, M.D. (2018). Assessing baseline and temporal changes in cardiometabolic risk using metabolic syndrome severity and common risk scores. Journal of the American Heart Association. 7(16):e009754. 10.1161/JAHA.118.009754. PMid:30369320

Gu, Y., \& Dennis, S. M. (2017). Are falls prevention programs effective at reducing the risk factors for falls in people with type-2 diabetes mellitus and peripheral neuropathy: A systematic review with narrative synthesis. Journal of Diabetes and its Complications. $31(2)$, 504-516. 10.1016/j.jdiacomp.2016.10.004

IBGE - Instituto Brasileiro de Geografia e Estatística. (2021). IBGE cidades. Rio Branco. http://www.ibge.gov.br/home/estatistica/populacao/censo/tabelas_pdf/total_populacao_acre.pdf

Kehoe, L., Walton, J., \& Flynn, A. (2019). Nutritional challenges for older adults in Europe: current status and future directions. Proceedings of the Nutrition Society. 78(2), 221-233. 10.1017/S0029665118002744

Lee, A. K., Juraschek, S. P., Windham, B. G., Lee, C. J., Sharrett, A. R., \& Coresh, J. (2020). Severe Hypoglycemia and Risk of Falls in Type 2 Diabetes: The Atherosclerosis Risk in Communities (ARIC) Study. Diabetes Care. 43(9), 2060-2065. 10.2337/dc20-0316

Liaw, F. Y., Kao, T.W., Wu, L. W., Wang, C. C., Yang, H. F., \& Peng, T. C. (2016). Components of Metabolic Syndrome and the Risk of Disability among the Elderly Population. International Journal of Scientific Reports. 10.1038/srep22750

Louvison, M., Lebrão, M., Duarte, Y., Santos, J., Malik, A., \& Almeida E. (2008). Desigualdades no uso e acesso aos serviços de saúde entre idosos do município de São Paulo. Rev. Saúde Pública. 42(4):733-40. 10.1590/S0034-89102008000400021

MS - Ministério da Saúde. (2020). Cartilha sobre a Situação de Hipertensão e Diabetes no Brasil. http://189.28.128.100/dab/docs/portaldab/docum entos/atlas_hipertensao_diabetes.pdf

Nascimento, M. (2017). Factores predictores de caídas en mujeres que practican actividad física. Rev. Brasileira em Promoção da Saúde. $30(3): 1-7$. $10.5020 / 18061230.2017 .6302$

Oliveira, E., Pereira, D., Ribeiro, E., \& Mukai, H. (2020). Prevalência de depressão em idosos com diabetes mellitus: uma revisão integrativa. CONICSEMESP. Retrieved May 22, 2021, from http://conic-semesp.org.br/anais/files/2020/trabalho-1000005635.pdf

Paiva, F., Lima, L., Funes, M., Volpe, C., Funguetto, S., \& Stival, M. (2019). A influência da dor na qualidade de vida de idosos portadores de Diabetes Mellitus / The influence of pain on elderly diabetics' quality of life / La influencia del dolor en la calidad de vida de ancianos portadores de Diabetes Mellitus. Rev. Enfermagem UERJ. 27: e31517. 10.12957/reuerj.2019.31517

Polit, D.F, \& Beck, C.T. (2019). Fundamentos de pesquisa em Enfermagem: Avaliação de evidências para a prática da Enfermagem. - 9.ed. - Porto Alegre: Artmed.

Prato, S., Andrade, S., Cabrera, M., Dip, R., Santos, H., \& Dellaroza, M. (2017). Frequência e fatores associados a quedas em adultos com 55 anos e mais. Rev Saúde Pública. 51:37. 10.1590/S1518-8787.2017051005409

Rinkel, W. D., Nieuwkasteele, S. V., Cabezas, M.C., Neck, J. W., Birnie, E., \& Coert, J. H. (2019). Balance, risk of falls, risk factors and fall-related costs in individuals with diabetes. Journal Diabetes Research and Clinical Practice. 10.1016/j.diabres.2019.107930

Shlisky, J., Bloom D. E., Beaudreault, A. R., Tucker, K. L., \& Keller, H. H. (2017). Nutritional Considerations for Healthy Aging and Reduction in AgeRelated Chronic Disease. Advances in Nutrition. 8(1), 17-26. 10.3945/an.116.013474

Silva, P., Sacramento, A., Carmo, I., Silva, L., Siqueira, S., \& Soares, S. (2019). Factors associated with metabolic syndrome in older adults: a populationbased study. Rev. Brasileira de Enfermagem. 22. (2), 228. 10.1590/0034-7167-2018-0620

Simão, A.F., Precoma, D.B., Andrade, J.P., Correa Filho, H., Saraiva, J.F.K., \& Oliveira, G.M.M. (2013). I Diretriz brasileira de prevenção cardiovascular. Arq. Brasileiros de Cardiologia.101(6),1-63. 10.5935/abc.2013S012.

Siqueira, F., Fachini, L., Silveira, D., Piccini, R., Tomas, E., \& Thumé, E. (2011). Prevalence of falls in elderly in Brazil: a contrywide analysis. Cad. Saúde Pública. 27(9):1819-1826. 10.1590/s0102-311×2011000900015 
Research, Society and Development, v. 10, n. 13. e483101320940, 2021

(CC BY 4.0) | ISSN 2525-3409 | DOI: http://dx.doi.org/10.33448/rsd-v10i13.20940

Sousa, N., Lima, M., Cesar, C., \& Barros, M. (2018). Active aging: prevalence and gender and age differences in a population-based study. Cad. Saúde Pública. 34(11):e00173317. 10.1590/0102-311x00173317

Tamura, Y., Omura, T., Toyoshima, K., \& Araki, A. (2020) Nutrition Management in Older Adults with Diabetes: A Review on the Importance of Shifting Prevention Strategies from Metabolic Syndrome to Frailty. Nutrients. 12(11), 3367. 10.3390/nu12113367

Vaupel, J.W., Villavicencio F., \& Bergeron-Boucher, M.P. (2021). Demographic perspectives on the rise of longevity. Proceedings of the National Academy of Sciences of the United States of America. 10.1073/pnas.2019536118. PMID: 33571137; PMCID: PMC7936303.

Wallander, M., Axelsson, K. F., Nilsson, A. G., Lundh, D., \& Lorentzon, M. (2017). Type 2 Diabetes and Risk of Hip Fractures and Non-Skeletal Fall Injuries in the Elderly: A Study from the Fractures and Fall Injuries in the Elderly Cohort (FRAILCO). Journal of Bone and Mineral Research. 32(3), 449-460. $10.1002 / \mathrm{jbmr} .3002$

WHO. World Health Organization. (2017). Integrated care for older people: guidelines on community-level interventions to manage declines in intrinsic capacity. World Health Organization. https://apps.who.int/iris/handle/10665/258981

Xavier, P., \& Trindade, A. (2018). Avaliação do risco de queda e equilíbrio em mulheres no climatério. Rev Kairós-Gerontologia. 21(2), 155-170. $10.23925 / 2176-901 X .2018 v 21$ i2p155-170 